\title{
Pratique du terrain et expérience des textes : une perspective africaine
}

Vers un nouveau régime (éco)poétique?

\section{Xavier Garnier}

\section{OpenEdition}

\section{Journals}

Édition électronique

URL : https://journals.openedition.org/coma/4132

DOI : $10.4000 /$ coma.4132

ISSN : 2275-1742

Éditeur

Institut des textes \& manuscrits modernes (ITEM)

\section{Référence électronique}

Xavier Garnier, "Pratique du terrain et expérience des textes : une perspective africaine », Continents manuscrits [En ligne], 12 | 2019, mis en ligne le 15 septembre 2019, consulté le 14 janvier 2023. URL :

http://journals.openedition.org/coma/4132 ; DOI : https://doi.org/10.4000/coma.4132

Ce document a été généré automatiquement le 14 janvier 2023.

\section{(c) $($ () $\ominus$}

Creative Commons - Attribution - Pas d'Utilisation Commerciale - Pas de Modification 4.0 International - CC BY-NC-ND 4.0

https://creativecommons.org/licenses/by-nc-nd/4.0/ 


\title{
Pratique du terrain et expérience des textes : une perspective africaine
}

\author{
Vers un nouveau régime (éco)poétique?
}

\author{
Xavier Garnier
}

1 L'approche anthropologique des textes est-elle un moyen de faire advenir leur poéticité ? Après une longue période coloniale d'approche ethnographique des textes africains, y a-t-il encore un sens à la notion de terrain du point de vue des études littéraires en Afrique postcoloniale ? À condition de l'associer à l'expérience, la notion de terrain reste très utile pour envisager l'approche des textes littéraires africains dans la perspective d'une poétique véritablement décentrée, ou décolonisée, qui se réclame des lieux et de ce que l'on y éprouve.

2 L'approche pragmatiste de la littérature que propose Florent Coste dans Explore ${ }^{1}$ répond au souci de l'insérer à tous les niveaux de l'expérience humaine, voire au-delà. Il s'agit de faire sortir le geste critique de l'illusion de la modernité et le faire « atterrir », pour reprendre les mots de Bruno Latour ${ }^{2}$. Le dialogue que je voudrais ouvrir avec Florent Coste porte sur la nécessité de maintenir une approche poéticienne des textes, une attention aux formes qui serait non contradictoire avec la perspective pragmatiste. Plusieurs attaques sur le «close reading » et sur l'« explication de texte » émaillent Explore, alors que ce livre porte explicitement le souci d'une articulation des formes de vie et des formes littéraires. Il me semble important de ne pas renoncer à décrire les formes littéraires pour montrer comment celles-ci sont des démultiplicateurs de forces et renforcent notre puissance d'agir. Cela est particulièrement flagrant depuis l'Afrique, dans un continent qui s'est retrouvé, dès l'époque coloniale, en situation d'évidement énergétique de formes ramenées à la folklorisation, la gesticulation, etc. Le choix d'une perspective africaine est en outre intéressante pour notre propos car, dès l'époque coloniale, la question d'un voisinage entre formes littéraires et ethnologie a été posée et mise en discussion. 


\section{Le moment colonial : les textes pris au piège du terrain}

3 Un premier enjeu est celui du partage impérial oral/écrit qui a présidé à l'épisode colonial en Afrique. L'idée d'une décontextualisation intrinsèque au texte écrit et particulièrement à l'imprimé depuis la «modernité » est à réévaluer dans une perspective africaine. Les travaux de Jack Goody nous ont montré que dans la logique impériale, l'écrit était très fortement arrimé à l'appareil d'État et à sa bureaucratie. Dans la distribution du pouvoir en situation coloniale, ceux qui travaillent sur les arts de la performance, les "maitres de la parole ", sont très compatibles avec le surplomb impérial de la graphosphère. L'efficience de l'oralité au sein de chaque ethnie est hautement valorisée par les dispositifs impériaux. On attend de la parole dite «traditionnelle » qu'elle soit efficiente (voire directement fonctionnelle), à condition que cette efficience ne déborde pas le cadre posé par l'Empire d'une délimitation stricte de l'ethnie. Dans le territoire dogon tout est possible : les maîtres de la parole peuvent bouleverser l'ordre cosmique par le verbe, l'administration impériale en prendra note avec une admiration intéressée et bienveillante. Dans la logique impériale, chaque ethnie est assignée à son terrain et, une fois ce cadre posé, est invitée à faire un usage efficient de sa parole hautement « culturalisée ».

4 On comprend du coup la tentation pour les intellectuels colonisés (dits " évolués ») d'un retrait hors du terrain. L'enjeu est pour eux de sortir du local pour parler au monde... via la littérature. Leur véritable terrain sera le papier, le livre, la bibliothèque, le champ littéraire français. Naît à l'époque coloniale une littérature "d'évolués ", qui passe par le livre pour engager un dialogue direct, au mépris des barrières ethniques qui enferment les colonisés dans leurs terrains respectifs. Les écrivains africains de l'époque coloniale multiplient les signes de rattachement au monde des «Lettres »: ils recherchent un style "hyper-littéraire", ils se font prendre en photo dans leur bibliothèque, stylo en main, la tête penchée sur leurs manuscrits, etc. L'écriture est le moyen d'échapper à la réification impériale. Il s'agit d'inventer une littérature d'interpellation et de jouer la "modernité » du livre contre la traditionalisation de l'oralité. Le défi est de transférer dans l'écriture la captation de forces que supposait l'oralité.

5 Le personnage éponyme de Giambatista Viko ou le viol du discours africain, le roman de l'écrivain congolais Georges Ngal, est un intellectuel accusé par les Anciens d'avoir mis le savoir traditionnel à disposition de l'archivistique coloniale, mais ce que le roman montre est que les accusateurs ont rendu possible la trahison par la mise en place d'un dispositif de cryptage. Tenir caché le savoir ethnique c'est jouer le jeu de l'Empire en maintenant la fiction d'une mystérieuse profondeur culturelle des dominés. L'universitaire du roman de Georges Ngal envisage au lieu de cela un espace ouvert pour une littérature gestuelle :

L'espace acoustique ou plus exactement audiovisuel. Celui du conteur! [...] Quelle

liberté dans l'évolution du récit! Aucune rigidité pareille à celle du roman!

Véritable cercle infernal, l'espace romanesque! Je rêve d'un roman sur le modèle

du conte! D'un roman où l'opposition entre diachronie et synchronie s'estompent :

où coexistent des éléments d'âge différent. D'un univers cinétique : qui engendre un ordre et s'engendre de lui ${ }^{3}$.

6 Il ne s'agit pas d'encrypter le conte dans le roman, ni même simplement de donner au roman la forme d'un conte, mais d'inscrire le roman dans l'espace du conte, ou plus exactement dans son "univers cinétique ». Giambatista Viko a l'ambition de faire 
sauter les cadres impériaux, d'emporter la bureaucratie impériale dans l'univers cinétique né de l'oral.

Dès lors que l'ambition pour les écrivains est de parler au monde, un nouveau problème est alors de ne pas se retrouver enfermé dans la sous-catégorie dévalorisée de la littérature coloniale, tenue en marge de tout champ littéraire. Comment faire résonner les œuvres? À l'approche des Indépendances, dans le contexte mondial propice de l'après-guerre, va s'inventer une pratique littéraire permanente de l'événement, dont «l'Indépendance » sera le point de focalisation.

\section{Moment panafricain : le terrain pris dans l'événement artistique}

8 Le moment fort du panafricanisme que furent les années soixante passe par la valorisation de l'œuvre comme événement. D'une certaine façon toute pratique fait alors œuvre: l'Afrique est artistique, «black is beautiful», tout est susceptible d'inventaire pour une Négritude triomphante. Loin d'enchâsser l'œuvre, le contexte est entraîné par l'événement! Dans l'enthousiasme incroyable des années soixante, l'Afrique ne cesse de se réinventer via les festivals, les événements musicaux, sportifs... mais aussi littéraires. Les littératures africaines font partie de ce que Bernard Mouralis appelle en 1975 les « contre-littératures », c'est-à-dire des littératures marginales, voire invisibles, qui tirent de leur marginalité une remarquable énergie performative.

9 À propos du cinéma de Jean Rouch, Deleuze dit de la fabulation qu'elle est une arme puissante pour le pauvre ou l'opprimé :

Ce qui s'oppose à la fiction, ce n'est pas le réel, ce n'est pas la vérité qui est toujours celle des maîtres ou des colonisateurs, c'est la fonction fabulatrice des pauvres, en tant qu'elle donne au faux la puissance qui en fait une mémoire, une légende, un monstre. [...] Ce que le cinéma doit saisir, ce n'est pas l'identité d'un personnage, réel ou fictif, à travers ses aspects objectifs et subjectifs. C'est le devenir du personnage réel quand il se met lui-même à «fictionner ", quand il entre «en flagrant délit de légender », et contribue ainsi à l'invention de son peuple"

Il s'agit de trouver un style "Indépendance-chacha», de faire de la littérature congolaise une littérature de "sapeurs », susceptible de faire basculer le réel à la pointe d'une phrase ou d'une élégance. Ahmadou Kourouma, qui publie Les Soleils des indépendances en 1968, invente un style événementiel, qui porte en boutonnière le malinké et renonce à une certaine image du beau style français, méthodiquement véhiculée dans les colonies.

11 Bakhtine écrit que les enjeux stylistiques sont associés à un "milieu vivant et concret »: c'est à partir de là que nous pouvons travailler pour définir l'approche écopoétique. On est très proche de ce que dit Barthes à propos du Texte et de sa puissance de modification du contexte. Le milieu n'est donc pas un préalable à l'étude du texte, il est mis en jeu par celui-ci, notamment dans sa dimension stylistique. Le milieu n'est donc pas une affaire de données à reconstituer en amont du texte, il est pris dans la dynamique de stylisation du texte. Dans la perspective écopoétique, le texte n'est pas produit par le contexte, il en est une puissance de métamorphose.

12 La quête du "style ", qui travaille la littérature africaine depuis l'époque coloniale, s'associe dans les années soixante à l'exigence de créer l'événement. S'ouvre le temps des parades postcoloniales. Les Black Panthers portent la révolution par leurs postures, 
les "écrivains-sapeurs ${ }^{5}$ » de la phratrie congolaise inscrivent cette révolution dans leurs manuscrits, le Shrine de Fela Ransome Kuti à Lagos dans les années soixante-dix est une première forme de ZAD au cœur du tissu urbain. Même pilotés par des États postcoloniaux pris dans la tenaille de la Guerre froide, les grands festivals panafricains de Dakar (1966) et d'Alger (1969), vibrent de cette ferveur événementielle qu'une analyse rétrospective des grands enjeux géostratégiques de l'époque ne saurait réduire.

\section{Le moment afropolitain ou l'ingénierie de l'événementiel}

13 Dans Culture et impérialisme, Edward Saïd a analysé les métamorphoses de l'Empire en réponse à cette offensive de l'émancipation par l'événement. À partir des années soixante-dix, les pouvoirs impériaux qui ont gardé le contrôle économique, sont en train de perdre l'hégémonie culturelle qui leur a permis de légitimer la conquête. Saïd explique que les références aux grands artistes et écrivains qui ont fait la culture occidentale n'ont plus besoin d'être convoquées pour justifier la supériorité de la Civilisation. Le nouvel Empire mondial s'appuie sur des experts et sur la maîtrise des canaux de l'information à échelle globale. En découle une façon très ambiguë d'articuler le local et le global.

14 L'ingénierie de l'événementiel va se substituer à l'hégémonie culturelle. L'UNESCO, l'OIF (avec l'Agence de coopération culturelle et technique) sont des plates-formes à échelle planétaire d'assistance technique pour la préservation, la prospection et la promotion de talents, etc. Des festivals ou des manifestations comme «Étonnants voyageurs" (manifestation basée à Saint-Malo mais régulièrement délocalisée sur d'autres continents), « Les Ateliers de la pensée » (qui se tiennent à Dakar) apparaissent comme l'aboutissement d'un phénomène qui a commencé au cours des années soixante-dix avec l'organisation à Kinshasa de la rencontre sur un ring de boxe entre George Foreman et Mohamed Ali.

15 L'organisation locale d'événements globaux n'est pas étrangère aux discussions sur le mouvement "afropolitain » qui, sans se réduire à cela, apparaît comme héritier de cette ingénierie globale d'événements culturels dont on choisit stratégiquement (ou géopolitiquement) la localisation. L'afropolitanisme est une notion complexe associée à la circulation globale des livres et des énoncés. Cette dimension circulatoire de l'afropolitanisme distingue ce mouvement du panafricanisme dont l'ambition était de constituer un môle bien ancré pour perturber une utopie littéraire trop peu consciente de la question raciale et des souffrances générées par la « ligne de couleur».

On en arrive à la revendication de désancrage maximum de la part d'un certain nombre d'écrivains africains contemporains, qui ne cessent cependant de se référer à l'Afrique dans leurs textes comme à un simple effet textuel, ou un signifiant flottant pris dans une série de mises en discours. Le terrain africain est perçu comme ce que le géographe Michel Lussault appelle un hyper-lieu, d'autant plus pertinent qu'il est hyper-connecté au global, capté par les caméras d'une presse-trash, quadrillé par les ONG humanitaires, sous perfusion des agences de transfert d'argent, etc.

17 La littérature africaine, arrimée au global, est volontiers qualifiée de "glocale » dès lors qu'elle se réfère à des lieux précis du continent, mais dans un geste tellement décentré 
qu'on peut légitimement se demander quelle métamorphose de l'expérience du lieu est alors engagée.

\section{Plaidoyer pour un moment de contre-offensive écopoétique}

18 À l'ingénierie globalisée de l'événement local, nous pourrions opposer l'expérience des lieux (ou des «hypo-lieux» pour trouver un répondant aux hyper-lieux de Lussault) comme arme contre la mondialisation financière et ses stratégies culturelles. L'enjeu est de libérer le local de sa dépendance à une pensée d'un global hyper-visibilisé, dont le web est la manifestation la plus exemplaire. Dans l'ouvrage collectif Africa's hidden histories: everyday literacy and making the self ${ }^{6}$, Karin Barber s'intéresse aux textes conservés dans des malles au sein des familles en Afrique centrale à l'époque coloniale (« littérature de cantine »). Cette littérature cachée peut être considérée comme une réponse obscure aux logiques de réification impériales. L'être du terrier de la célèbre nouvelle de Kafka, qui creuse et habite ses galeries souterraines, est une figure de l'écrivain en situation impériale. Sa littérature est tout aussi monumentale que les tours d'ivoire, mais ses monuments sont souterrains, ils ne sont pas faits pour être contemplés de l'extérieur, mais pour être pénétrés ${ }^{7}$. On entre dans les galeries comme on suit les configurations des textes, pour faire résonner le monde et émettre des voix inassignables.

Si la bibliothèque est une hétérotopie dans le célèbre article de Michel Foucault, elle s'est déversée dans l'espace quotidien de beaucoup de villes africaines et c'est là qu'il faut aller chercher les textes. Il y a dès lors toute une réflexion à mener sur la question de l'inscription du texte, qui rejoint de nombreuses préoccupations actuelles sur les supports de la littérature : il existe un terrain pour les textes dont le livre n'est qu'une modalité. Écrire ou lire c'est aussi faire l'expérience d'un support pour l'écrit, particulièrement dans des parties du monde où le(s) papier(s) parle(nt) la langue du pouvoir. Dans Discours/figure, Jean-François Lyotard s'intéresse au substrat figural de tout discours et la façon dont il se rattache à son support. Il y a continuité entre un poème griffonné sur un mur à Libreville et un poème inséré dans un livre rangé dans une bibliothèque à Paris.

Il ne faut pas pour autant renoncer à considérer la bibliothèque occidentale comme un monument. Il y a une analyse stylistique possible à faire des textes écrits sur les camions, sur les pagnes, sur les devantures, qui devra rendre compte des configurations qu'ils nous proposent. Ces œuvres disséminées nous apprennent à voir les textes comme des milieux où il est possible de faire une expérience, comme Roland Barthes nous invite à le faire :

[...] le lecteur du Texte pourrait être comparé à un sujet désœuvré (qui aurait détendu en lui tout imaginaire) : ce sujet passablement vide se promène (c'est ce qui est arrivé à l'auteur de ces lignes, et c'est là qu'il a pris un idée vive du Texte) au flanc d'une vallée au bas de laquelle coule un oued (l'oued est mis là pour attester un certain dépaysement); ce qu'il perçoit est multiple, irréductible, provenant de substances et de plans hétérogènes, décrochés: lumières, couleurs, végétations, chaleur, air, explosions ténues de bruits, minces cris d'oiseaux, voix d'enfants de l'autre côté de la vallée, passages, gestes, vêtements d'habitants tout près ou très loin; tous ces incidents sont à demi identifiables : ils proviennent de codes connus, 
mais leur combinaison est unique, fonde la promenade en différence qui ne pourra

se répéter que comme différence ${ }^{8}$.

21 Éprouver le texte, c'est reconnaître ses dénivelés, ses pentes, ses décrochages. Il est important de pénétrer dans le texte, car c'est la seule façon de l'éprouver. Ce n'est pas le texte qui circule dans le monde (si l'on pose le problème ainsi on bute sur les enjeux d'ingénierie), mais nous qui sommes invités à circuler dans le texte pour voir le monde depuis un lieu «irréductible ». L'irréductibilité du lieu tient à la façon dont il a été creusé dans le territoire. Le livre, l'écran, au même titre que tout support scriptural, sont des trous dans le territoire qui permettent d'accéder au terrier comme caisse de résonance du monde.

En termes écologiques, l'enjeu est de sortir la littérature de sa tour d'ivoire pour la faire rentrer dans la zone critique, cette bande étroite autour de la surface de la planète où se joue la survie de nombreuses espèces.

\section{BIBLIOGRAPHIE}

Barber, Karin (éd.), Africa's hidden histories: everyday literacy and making the self, Bloomington, Indiana University Press, 2006.

Barber, Karin, The Anthropology of texts, persons and publics. Oral and written culture in Africa and beyond, New-York, Cambridge University Press, 2007.

Barthes, Roland, « De l'œuvre au texte », Le Bruissement de la langue. Essais critiques IV, Paris, Le Seuil, 1993, p. 75.

Deleuze, Gilles, L'image-Temps. Cinéma 2, Paris, Minuit, 1985.

Foucault, Michel « Des espaces autres », Dits et écrits II. 1976-1988, Paris, Gallimard, Quarto, 2001, p. 1578 .

Ngal, Georges, Giambatista Viko ou le viol du discours africain, Paris, Hatier, 1970.

\section{ANNEXES}

\section{À propos de Explore de Florent Coste : chronique de Nathalie Quintane (extrait) :}

"Ceux qui ont connu le creux de la vague en matière de théorie littéraire et la panne de libido nationale qui laisse les amphis des facs de lettres exsangues et les chaises des festivals estivaux peuplées de têtes blanchies sous le harnois musso-houellebecquien ne peuvent qu'être soulagés (premier temps) puis excités (deuxième) par ce retour à présent acté de l'expérience littéraire.

Le livre de Florent Coste enfonce encore le clou et va plus loin : fin de l'enclave littéraire, c'est-à-dire fin de la clôture disciplinaire et fin du "concept" Littérature bordé de tous côtés, terminato le close reading solennel et le corpus sacré qu'on compte sur nos dix doigts (Balzac-Baudelaire-Barthes etc) : bref, fin de la course en sac. Rappelant la nécessité d'une alliance des Lettres avec d'autres secteurs disciplinaires (la 
part belle est faite à l'anthropologie), d'efforts théoriques centrés sur le lecteur plus que sur l'auteur, le fait qu'art et littérature sont des concepts empiriques, labiles, réformés à chaque œuvre aussi bien qu'à chaque lecture, Coste décrit avec précision, élan et conviction, ce que littérature et poésie nous font : un livre bon nous "réengage plus vifs" dans la vie, il "mobilise différents régimes d'attention" et "réveille nos capacités critiques". Il "nous installe à un poste d'exploration active" - d'où le titre du livre : Explore, organisé en 7 exercices, où l'adresse directe au lecteur tutoyé évoque les textes ardents et gais d'un Diderot (là aussi se joue la compréhension en acte dans la phrase même d'un nécessaire retour critique et combatif, r-appelé justement par Marielle Macé mais qui parfois stylistiquement semble faiblir dans son dernier livre). Coste ne voit pas la littérature "comme un réservoir plus ou moins privilégié d'informations dont on ferait un usage exclusivement documentaire", ce n'est donc pas une version pragmatique ou utilitariste qu'il nous propose, mais véritablement pragmatiste : la littérature façonne et forme des vies plus sensibles, plus incarnées, plus politiques. Qu'il y a une vie littéraire possible, comme il y eut des vies philosophiques, c'est ce qu'affirme cet essai enthousiasmant ».

\section{NOTES}

1. Florent Coste, Explore: investigations littéraires, Paris, Questions théoriques, coll. «Forbidden beach ", 2017. Cf. en annexe de cet article la chronique de Nathalie Quintane accessible à cette adresse : https://www.sitaudis.fr/Parutions/explore-de-florent-coste.php.

2. Bruno Latour, Où atterrir ?, Paris, La Découverte, coll. « Cahiers libres », 2017.

3. Georges Ngal, Giambatista Viko ou le viol du discours africain, Paris, Hatier, 1970, p. 10.

4. Gilles Deleuze, L'image-Temps. Cinéma 2, Paris, Minuit, 1985, p. 196.

5. L'anthropologue Patrice Yengo, lors d'une discussion au séminaire de l'équipe manuscrits francophones de l'ITEM, a comparé les écrivains de la phratrie congolaise dans les années soixante-dix à des sapeurs qui exerceraient leur activité dans le domaine littéraire.

6. Karin Barber (éd.), Africa's hidden histories: everyday literacy and making the self, Bloomington, Indiana University Press, 2006.

7. Le titre de la nouvelle de Kafka est Der Bau, c'est-à-dire « la construction ».

8. Roland Barthes, "De l'œuvre au texte », Le Bruissement de la langue. Essais critiques IV, Paris, Le Seuil, 1993, p. 75.

\section{RÉSUMÉS}

L'approche anthropologique des textes est-elle un moyen d'en faire advenir leur poéticité ? Telle est la question à laquelle j'essaie de répondre. Parce qu'elle sont à la croisée de l'anthropologie et

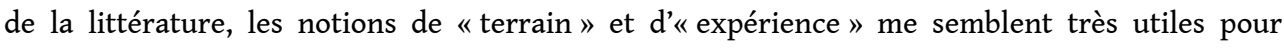
envisager l'approche des textes dits littéraires dans la perspective d'une poétique véritablement décentrée, ou décolonisée. 
INDEX

Mots-clés : terrain, expérience, éco-poétique, décentrement

\section{AUTEUR}

\section{XAVIER GARNIER}

Professeur de littérature française et francophone à l'Université Sorbonne Nouvelle-Paris 3 